\title{
A Tool for Modelling Business Behaviour Using Decision Tables
}

\author{
Tomas Antunes \\ Victoria University of \\ Wellington

\begin{abstract}
Pedro Antunes
Wellington
\end{abstract} \\ Victoria University of \\ Victoria University of \\ Wellington \\ Wellington, New Zealand Wellington, New Zealand Wellington, New Zealand \\ tomas.antunes@icloud.com pedro.antunes@vuw.ac.nz david.johnstone@vuw.ac.nz
}

\author{
Vo Trong Nghia \\ Can Tho University of \\ Technology \\ Can Tho, Vietnam \\ vtnghia.ktpm0115@student. \\ ctuet.edu.vn
}

\author{
Nguyen Hoang Thuan \\ Can Tho University of \\ Technology \\ Can Tho, Vietnam \\ nhthuan@,ctuet.edu.vn
}

\begin{abstract}
Decision tables have been recently recognised as an effective technique to model business behaviour. The increasing adoption of decision tables in this context requires appropriate end-user support. Although several tools have been developed using decision tables, few have been targeted to business end users. This paper fulfils this gap by proposing a tool supporting end users to capture and model business behaviour. The proposed tool integrates decision tables for capturing actionable knowledge using ontology technology. The tool is validated in two case studies.
\end{abstract}

Keywords- Decision Tables, Decision Tools, Ontologies, Camunda, Protégé, Case study.

\section{INTRODUCTION}

For decades, decision tables have been used to represent decisions in the context of programming logic [1]. Recently, the use of decision tables has gained momentum in the business context with the purpose to better model business behaviour. The fundamental goal is to improve business systems such as order management, service delivery and client relationship management, just to mention few that could benefit from improved business modelling. A contributing factor to the wider adoption of decision tables in the business context is the decision by the Object Management Group (OMG) to integrate decision tables into the Decision Model and Notation (DMN) standard, which is currently used by many business systems $[2,3]$.

Integrated with DMN, the approach enables system developers to create business systems that can be injected with business models defined by the end users themselves [3, 4]. Such approach has been named either end-used design or metadesign [5]: while developers define the meta functionality, end users finish the design with their contextualised business models.

Furthermore, decision tables are viewed as a more flexible way to model business behaviour, especially when compared to similar technology such as decision trees and oblique rules [6]. Decision tables are also considered easier to integrate with business systems, in particular business process management [7]. These important capabilities have motivated an increased research interest, as suggested by an increasing number of publications on the topic $[2-4,8]$.

By and large, the body of literature on decision tables embraces two very distinct problems, which can be related to computer science and information systems (IS). In the computer science domain, researchers are mainly interested in the formalisation, standardisation, automation, optimisation, and integration of decision tables at the technical level. Examples in this category include semantic analysis [7, 9-12], detection of inconsistencies and missing rules [7, 10, 11], rule formalisation, and optimisation [2].

On the other hand, the IS field is more concerned with the use, adoption and implementation of the technology at the business and organisational levels. Examples in this category include business modelling, decision making, knowledge integration, knowledge management, business support, and usability $[4,8,13]$.

This paper concerns business modelling from an IS perspective. In particular, we are interested in understanding how to support end users to meta design business behaviour through decision tables. This problem is challenging because of the distance between the technical and the organisational/end user levels. Such distance makes the approach more amenable to technology experts (e.g., developers, requirements engineers and technical analysts) than to end users (e.g. middle managers, business analysts and decision makers). This problem is relevant because a solution creates opportunities for developing more flexible, resilient and accessible business systems.

In particular, this research addresses the following goals:

1. Enable end users to model business behaviour using decision tables. Therefore, we research decision tables from an end user perspective;

2. Integrate decision tables with ontology technology. While decision tables provide actionable knowledge about business behaviour, ontological models provide the underlying definitional knowledge $[14,15]$;

3. Enable end users to explore what-if business scenarios by interacting with decision tables. Here, we note that business models often involve multiple factors and conflicting values, which require users to explore different possibilities before making an informed decision about the best course of action.

This study adopts a Design Science Research (DSR) paradigm, dividing the research in two main activities, build and evaluate [16]. Regarding the build activity, we use open source technology to integrate decision tables with ontology technology. In particular, we use Camunda [17] and Protégé [18] to model business behaviour. Camunda is used to manage decision tables, while Protégé is used to manage definitional 
knowledge. The solution allows end users to model business behaviour and perform what-if analysis.

Regarding the evaluate activity, we adopt the case study approach to illustrate the usefulness of the developed solution, considering in particular the three abovementioned research goals. The selected cases consider order discount and delivery [3], and business process crowdsourcing [15, 19].

This research provides the following contributions. Firstly, we develop a solution that supports end users in independently modelling business behaviour using ontologies and decision tables. Secondly, we illustrate the usefulness of the approach.

\section{LITERATURE REVIEW}

A decision table is a tabular structure for describing and analysing decisional logic, which defines what actions can be performed depending on given conditions [20]. While there are different ways to represent decision tables, they generally consist of three basic elements:

- Conditions: An input column defines alternative conditions, which are set or not depending on an independent variable. Table 1 presents an example of a decision table with two input columns considering customer and order size as variables.

- Actions: Output columns define which actions are executed after applying the corresponding rules. Table 1 shows a decision table with one output column named discount. The $5 \%$ action is executed if rule 3 is applied.

- Rules: Rows define rules linking specific conditions to actions. Rules are applied when the corresponding conditions are true. The decision table in Table 1 has three rules. Rule 3 is applied if a customer is private. The symbol (-) means the condition is true by default.

TABLE I. AN EXAMPLE OF A DECISION TABLE

\begin{tabular}{|l|l|c||c|}
\hline \multirow{2}{*}{ Rules } & \multicolumn{2}{|c||}{ Input } & Output \\
\cline { 2 - 4 } & Customer & Order size & Discount \\
\hline \hline 1 & Business & $>=10$ & $15 \%$ \\
\hline 2 & Business & $<10$ & $10 \%$ \\
\hline 3 & Private & - & $5 \%$ \\
\hline
\end{tabular}

We now review the literature on decision tables, focusing on the recent interest in using decision tables for business modelling $[2,4,8-10,21,22]$. Since the introduction of the DMN standard, several research lines have explored how to use this technology.

A research line has been centred on how to specify and validate business rules using decision tables, so they are complete, consistent and non-redundant $[2,7,8,10,11,13]$. Algorithms have been developed to detect overlaps, conflicts and missing rules $[7,10,11]$, and to simplify these tables [2, 23]. These studies extend prior research in computer science $[20,24]$, but applied to the more complex business context.
Several proof-of-concept tools have been developed to evaluate the proposed algorithms. Laurson and Maggi [10] developed a tool for verifying missing and overlapping rules in decision tables. In a similar vein, Batoulis and Weske [7,9] developed a tool that checks overlapping rules and simplifies decision tables.

Another research line is centred on how to model business behaviour using decision tables. The overarching goal is to integrate decision tables with business processes. This goal can be further decomposed into two related goals [4, 21, 22]: integrating business processes with the domain knowledge and business logic (conditions, actions and rules) expressed in decision tables; and automating the execution of business rules within the execution of business processes [10]. In particular, several proof-of-concept tools have been developed integrating business process modelling (e.g., BPMN) and decision modelling [21, 22, 25].

In our research, we adopt an alternative viewpoint. Instead of representing business behaviour using a combination of decision and process modelling, we consider a combination of decision and ontology modelling [4, 26, 27]. While ontologies provide definitional knowledge about a business $[14,15,26,27]$, decision tables provide actionable knowledge about the business behaviour.

However, little attention has been given in the related literature to the integration of definitional and actionable knowledge, considering in particular the integration of ontologies and decision tables as a way to represent business behaviour. This paper aims to address this research gap.

\section{METHOD}

Our research adopts the DSR approach to develop a proofof-concept tool. The approach comprises two activities: build and evaluate [16]. Fig. 1 and the text below describe the two activities in more detail.

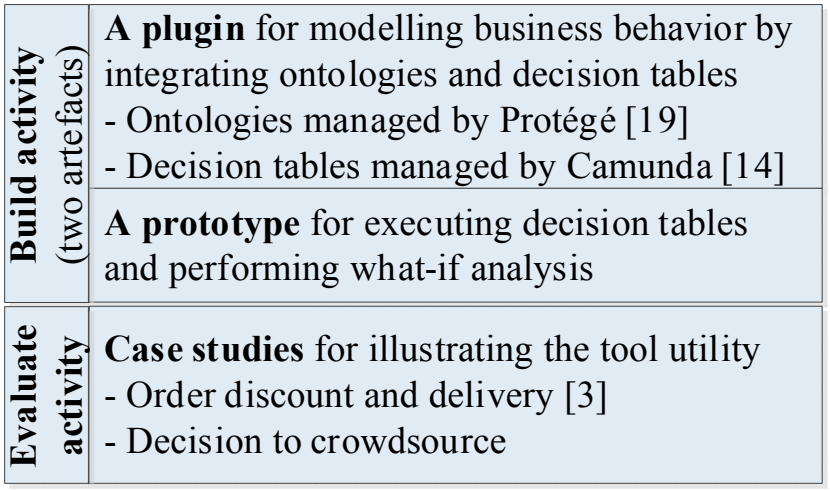

Fig. 1. Research activities

The build activity aims to develop a proof-of-concept tool modelling business behaviour using decision tables. The tool consists of two artefacts. The first artefact is a plugin allowing end users to model business behaviour using an ontology and several decision tables. The second artefact is a visualiser allowing end users to explore the model in what-if scenarios. 
We evaluate the proof-of-concept tool in two case studies. We purposefully chose two cases with different characteristics. The first case - order discount and delivery - is adapted from DMN [3] and illustrates that the tool is suitable for making business operational decisions. The second case - decision to crowdsource - is more related to business strategy [19], as it supports end users making decisions about crowdsourcing depending on several contingency factors.

\section{ToOl DeVELoPMent}

\section{A. The Plugin}

The main purpose of the plugin is to model business behaviour. We used existing open-source software to develop the plugin. More specifically, we rely on Camunda to manage decision tables and Protégé to manage ontologies. The plugin publicly is available at:

\section{https://github.com/tom277/EBM ruleManagement}

The plugin consists of ontology and decision modules. The ontology module is shown on the left-hand side of Fig. 2 . Empowered by Protégé, it enables end users to define classes, instances, attributes, and relations, which together provide definitional knowledge about a business. The case shown in Fig. 2 shows that the business behaviour considers orders, deliveries and discounts.

The decision module is shown on the right-hand side of Fig. 2. It enables end users to create and link decision tables to particular concepts defined in the ontology. For instance, Fig. 2 shows that discount rules have been defined and linked to the discount concept. Decision tables are defined using Camunda.

\section{B. Visualiser}

The visualiser enables end users to explore what-if scenarios of business behaviour using the models defined with the plugin (see Fig. 5 and Fig. 6). The visualiser was developed in Java and is available at:

\section{https:/github.com/tom277/EBM tool}

The visualiser consists of two main modules: ontology visualisation and decision visualisation. The ontology is visualised as a tree structure, reflecting the typical hierarchical structure of ontological representations. It provides an overview of the concepts defined in the business domain. Users can select the elements to see definitions and relationships to other elements.

When the end user selects a decision element, the visualiser shows the related decision tables. The decision visualisation module highlights a set of decision conditions that the user may change by configuring input variables. When the user configures an input, the module executes the decision rules specified in the decision tables and visualizes the outputs. This allows end users to perform what-if analysis by changing the inputs and analysing the outputs.

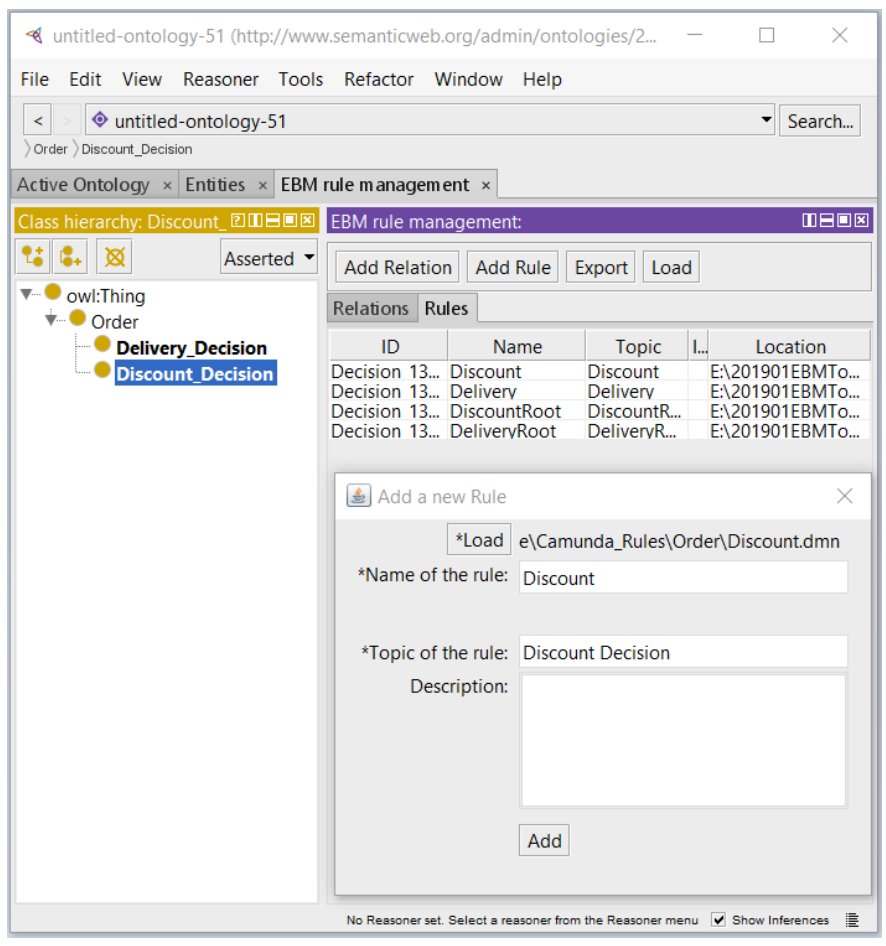

Fig. 2. Plugin integrating ontologies and decision tables

\section{CASE STUdY}

We describe two case studies validating the proof-ofconcept tool. For each case, we provide background information and discuss how the tool models business behaviour.

\section{A. Order Discount and Delivery (adapted from [3])}

When company X processes customers' orders, it has to make two relevant decisions. The first is to decide how much discount a particular order gets, which depends on two factors: type of customers (business vs. private) and order size ( $>=10$ vs. $<10$ ). The second is to decide how to deliver the order, which depends on the order's weight.

The decision to make a discount includes three business rules. Camunda is used to create the decision table shown in Fig. 3.

\begin{tabular}{|c|c|c|c|}
\hline \multicolumn{2}{|r|}{ Discount } & & \\
\hline \multicolumn{4}{|c|}{ Decision_13nychf } \\
\hline \multirow[t]{3}{*}{$\mathbf{F}$} & \multicolumn{2}{|c|}{ Input + } & Output + \\
\hline & Customer: Business vs. Private & Order size & Discount \\
\hline & string & string & string \\
\hline 1 & "Business" & ">=10" & "0.15" \\
\hline 2 & "Business" & $"<10 "$ & "0.10" \\
\hline 3 & "Private" & - & "0.05" \\
\hline
\end{tabular}

Fig. 3. Decision table: Discount

The delivery decision includes two business rules. Fig. 4 shows the decision table capturing these rules. 


\begin{tabular}{|c|c|c|}
\hline & Delivery & \\
\hline Dec & ision_13nychf & \\
\hline $\mathbf{F}$ & Input + & Output + \\
\hline & Weight & Delivery \\
\hline & string & string \\
\hline 1 & $"<=10 \mathrm{Kg} "$ & "by air" \\
\hline 2 & ">10 Kg" & "By ship" \\
\hline
\end{tabular}

Fig. 4. Decision table: Delivery

The plugin is then used to model business behaviour, which includes definitional and actionable knowledge. Regarding definitional knowledge, we model three basic concepts: order, discount decision, and delivery decision. Order is defined as the parent concept, and the others are sub-concepts. We then relate these concepts to the two decision tables previously discussed: the discount decision table is added to the discount concept; and the delivery decision table is added to the delivery concept (see Fig. 2).

The visualiser can then used to display the definitional and actionable knowledge, and to analyse what-if scenarios (Fig. 5). The visualiser shows the three business concepts (order, discount decision), including parent-child relationships, and associated rules. When the end user selects a concept, the associated decision rules are visualised. The end user can then configure different options and analyse the outputs. When the end user changes the configuration, the visualiser combines the new inputs with the actionable knowledge and generates the corresponding decisions. The visualiser generates the business behaviour automatically, so that the end user can analyse the results immediately. For instance, if the user changes the order size from " $>=10$ " to " $<10$ ", the visualiser will immediately suggest 0.10 as the new discount.

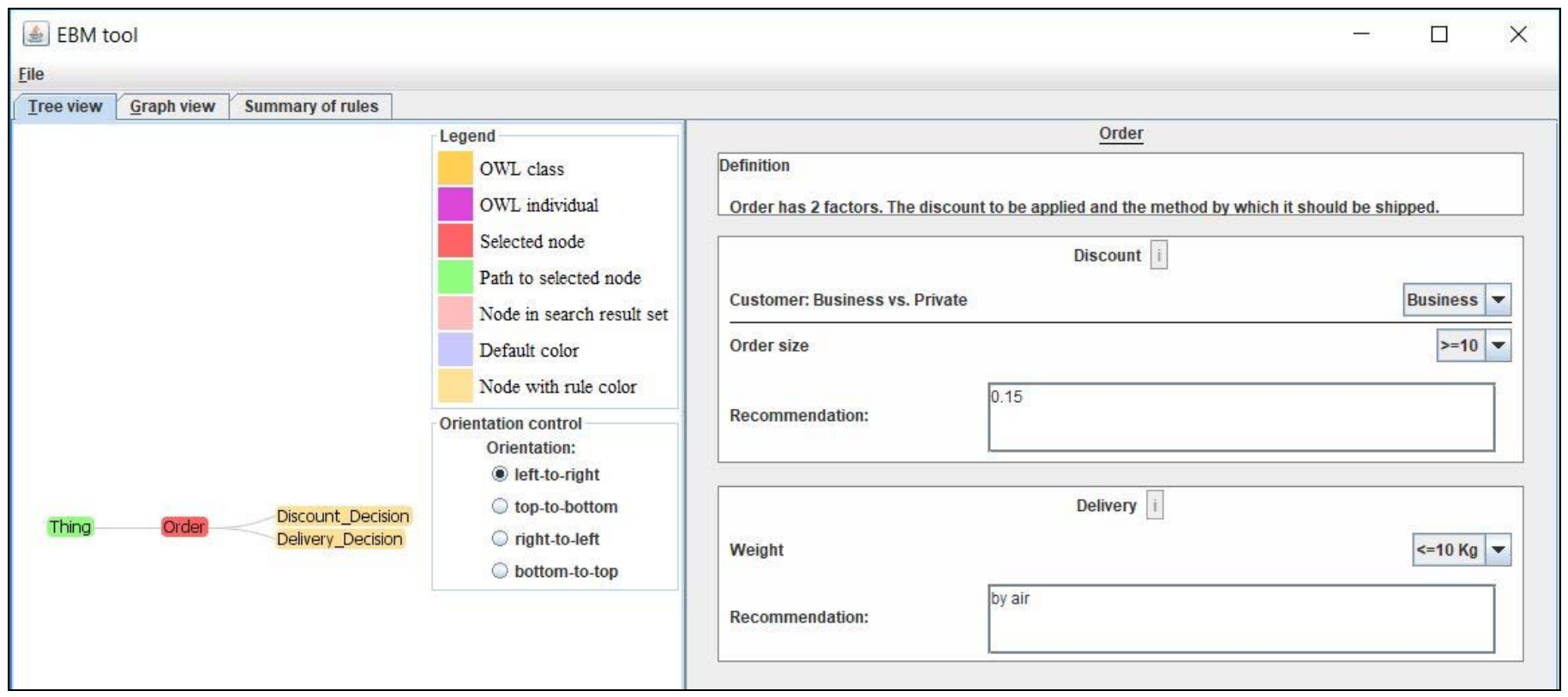

Fig. 5. Visualiser for the order discount and delivery case

\section{B. Decision to Crowdsource}

Crowdsourcing is an emerging way to use an online workforce to outsource business tasks. The decision to crowdsource is an important decision for organizations to strategize how to use such workforce. Yet, it is a complex decision for organisations, since several contingency factors should be examined [19, 28]. Seventeen relevant decision factors influencing the decision to crowdsource have been identified [19, 28]. Based on these factors, several decision tables can be defined, regarding different aspects of crowdsourcing such as task properties, people, management, and environment [19].

Furthermore, the decision factors have to be considered along with other defining aspects of the organization, which concern definitional knowledge. The decision to crowdsource is therefore complementary to definitional knowledge, which has been specified using an BPC ontology [15].
Using our proof-of-concept tool, we can support businesses making the decision to crowdsource. The plugin is used to model the BPC concepts, their hierarchical relationships, as well as the decision rules. The visualiser is then used to analyse different scenarios and decide what to do. Fig. 6 shows the visualiser when making the decision to crowdsource.

By using this tool, end users can model the crowdsourcing behaviour and perform what-if analysis of the available alternatives. The left-hand side of Fig. 6 shows the definitional knowledge required to make the decision to crowdsource, while the right-hand side shows the action possibilities. The visualiser shows the definitional knowledge as a graph, which can be navigated. The end user can navigate the ontological concepts related to crowdsourcing, including definitions, references, relationships, and can link the concepts to actionable knowledge.

When the end user accesses a concept referring to business decisions, the visualiser shows the relevant decision tables (the 
right-hand side of Fig. 6). In the example illustrated in Fig. 6, the visualiser shows four decision tables regarding the decision to crowdsource, which enables users to execute various related business rules.

We note that making the decision to crowdsource is not straightforward but needs to consider multiple factors. The visualiser enables end users to change the input data and observe the outputs. As a result, the user gains a better understanding of the decision to crowdsource. The 'Summary of Rules' tab provided by the visualizer allows end users to review decisions arising from crowdsourcing rules. This tab provides knowledge required to make an informed decision.

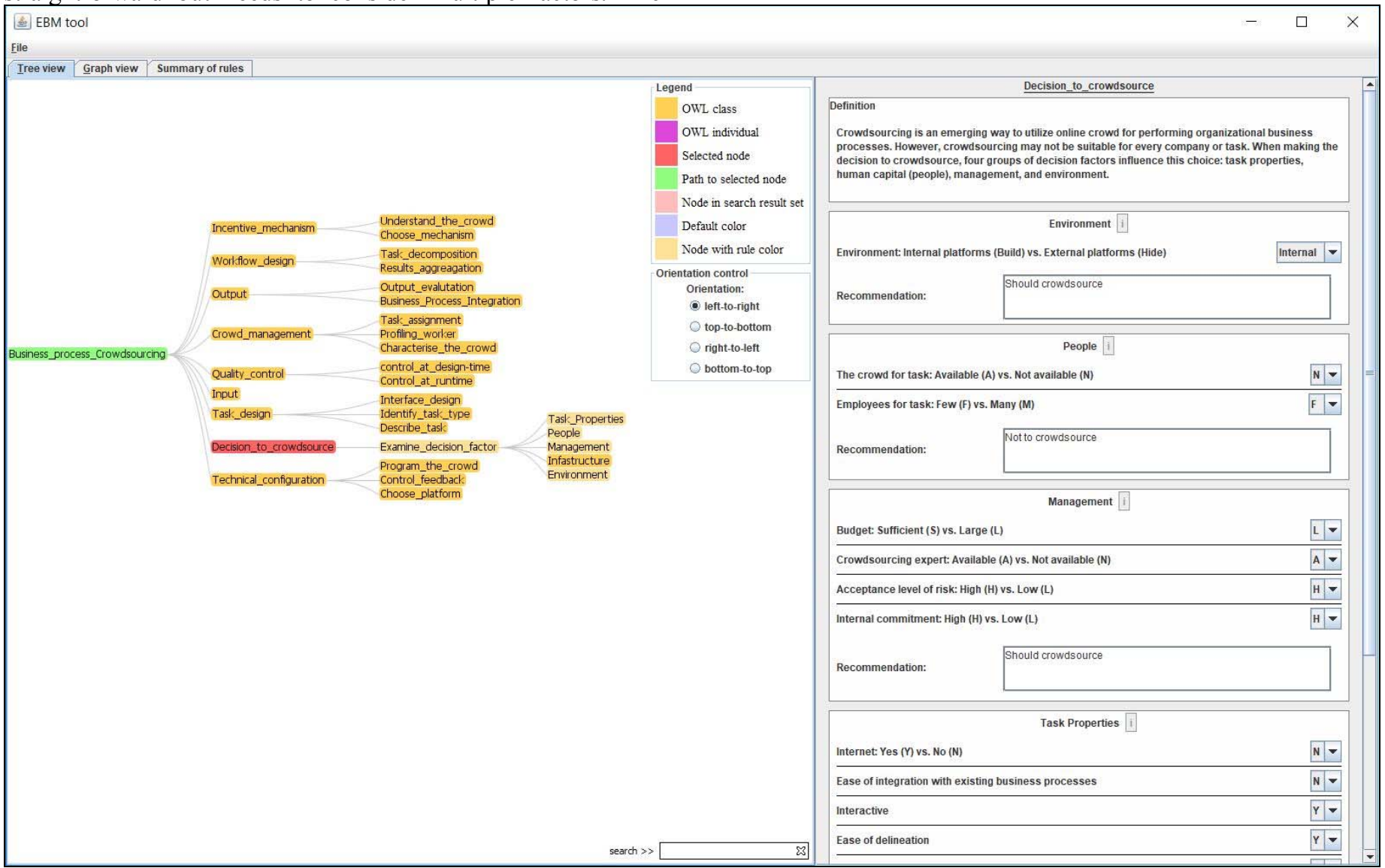

Fig. 6. Visualiser for the decision to crowdsource case

Overall, the two cases show how the tool supports end users modelling business behaviour and making decisions. The first case, which has been adapted from DMN, shows the tool is suitable for making business decisions. The second case shows the tool can be used to explore complex decisions. Both cases highlight the tool can manage ontological and actionable knowledge. They also highlight how the tool can support whatif analysis to make more informed decisions.

\section{DISCUSSION AND CONCLUSION}

This study assesses the adoption of decision tables to model business behaviour $[4,8,13]$. We developed a proof-ofconcept tool that supports end users defining business behaviour and making what-if decisions about which decisions to make in specific business contexts. The tool inherits the strengths of decision tables and ontology technologies. While ontologies capture definitional knowledge [26, 27, 29], decision tables capture actionable knowledge [4]. We integrated the two technologies in a tool that is targeted to end users. The tool was used in two business cases. The cases illustrate how the tool can be used by end users to create, model, visualise, and make decisions depending on the business context.

This study contributes to business modelling and knowledge management. We integrate definitional and actionable knowledge using ontologies and decision tables. The approach contrasts with prior research where business behaviour is modelled using a combination of business processes and decision tables [21, 22, 25]. Our work suggests that definitional knowledge is an important element in business modelling. Furthermore, we suggest that end users can model and explore business behaviour using what-if scenarios.

Ontologies have been applied to formalise and share knowledge $[14,15]$. This study extends the use of ontologies to behaviour modelling in business contexts. We suggest the integration of decision tables and ontologies in business systems, so they can inform decision making. We regard the integration of decision tables and ontologies as exaptation [30]: using well-known solutions to address new problems. 
Furthermore, we suggest our approach can be adopted to meta design business systems: allowing end users to finish the design of business systems by defining the models and rules governing business behaviour.

From a more practical perspective, the paper provides a proof-of-concept tool supporting future empirical research. The discussed cases show how the tool can be used in business environments to make business decisions based on what-if analysis.

However, this study has certain limitations. Firstly, while the tool uses Protégé to represent concepts, attributes, and relations, we understand that Protégé has additional features including formalisation, axioms, and queries, which have not been used. We will continue to explore these features in modelling business behaviour. Secondly, the tool was developed for research purposes, not actual use by organisations. Future research should bring actual end users into the design process. Finally, while we have illustrated the tool's usefulness in two cases, further evaluation is necessary to evaluate the tool.

\section{REFERENCES}

[1] Kirk, H.W.: 'Use of decision tables in computer programming', Communications of the ACM, 1965, 8, (1), pp. 41-43

[2] Calvanese, D., Dumas, M., Laurson, Ü., Maggi, F.M., Montali, M., and Teinemaa, I.: 'Semantics, analysis and simplification of DMN decision tables', Information Systems, 2018, 78, pp. 112-125

[3] OMG: 'Decision Model and Notation (version 1.2)', Object Management Group Specification, 2019

[4] Deryck, M., Hasić, F., Vanthienen, J., and Vennekens, J.: 'A CaseBased Inquiry into the Decision Model and Notation (DMN) and the Knowledge Base (KB) Paradigm', 'Book A Case-Based Inquiry into the Decision Model and Notation (DMN) and the Knowledge Base (KB) Paradigm' (Springer, 2018, edn.), pp. 248-263

[5] Fischer, G., Giaccardi, E., Ye, Y., Sutcliffe, A.G., and Mehandjiev, N.: 'Meta-design: a manifesto for end-user development', Communications of the ACM, 2004, 47, (9), pp. 33-37

[6] Huysmans, J., Dejaeger, K., Mues, C., Vanthienen, J., and Baesens, B.: 'An empirical evaluation of the comprehensibility of decision table, tree and rule based predictive models', Decision Support Systems, 2011, 51, (1), pp. 141-154

[7] Batoulis, K., and Weske, M.: 'A Tool for Checking Soundness of Decision-Aware Business Processes', 'Book A Tool for Checking Soundness of Decision-Aware Business Processes' (2017, edn.), pp. 1-5

[8] Calvanese, D., Dumas, M., Maggi, F.M., and Montali, M.: 'Semantic DMN: Formalizing decision models with domain knowledge', 'Book Semantic DMN: Formalizing decision models with domain knowledge' (Springer, 2017, edn.), pp. 70-86

[9] Batoulis, K., and Weske, M.: 'A Tool for the Uniqueification of DMN Decision Tables', Proceedings of the Dessertation Award and Demonstration, 2018, pp. 1-4

[10] Laurson, Ü., and Maggi, F.M.: 'A Tool for the Analysis of DMN Decision Tables', BPM 2016 Demonstration Track, 2016, pp. 56-60

[11] Vanthienen, J., and Dries, E.: 'Illustration of a decision table tool for specifying and implementing knowledge based systems', International Journal on Artificial Intelligence Tools, 1994, 3, (2), pp. 267-288
[12] Corea, C., and Delfmann, P.: 'A Tool to Monitor Consistent DecisionMaking in Business Process Execution', Proceedings of the Dissertation Award, Demonstration, and Industrial Track at BPM, 2018, pp. 9-14

[13] Demey, Y.T., and Tran, T.-K.: 'Using SOIQ(D) to Formalize Semantics within a Semantic Decision Table', 'Book Using SOIQ(D) to Formalize Semantics within a Semantic Decision Table' (Springer, 2012, edn.), pp. 224-239

[14] Gruber, T.R.: 'A translation approach to portable ontology specifications', Knowledge acquisition, 1993, 5, (2), pp. 199-220

[15] Thuan, N.H.: 'Business Process Crowdsourcing: Concept, Ontology and Decision Support' (Springer, 2019. 2019)

[16] Hevner, A., March, S.T., Park, J., and Ram, S.: 'Design science in information systems research', MIS Quarterly, 2004, 28, (1), pp. 75-105

[17] Camunda: 'Decision Automation', 2019, https://docs.camunda.org/getstarted/quick-start/decision-automation/

[18] Gennari, J.H., Musen, M.A., Fergerson, R.W., Grosso, W.E., Crubézy, M., Eriksson, H., Noy, N.F., and Tu, S.W.: 'The evolution of Protégé: an environment for knowledge-based systems development', International Journal of Human-computer studies, 2003, 58, (1), pp. 89123

[19] Thuan, N.H., Antunes, P., and Johnstone, D.: 'Factors Influencing the Decision to Crowdsource: A Systematic Literature Review', Information Systems Frontiers, 2016, 18, (1), pp. 47-68

[20] Vanthienen, J., and Wets, G.: 'From decision tables to expert system shells', Data \& Knowledge Engineering, 1994, 13, (3), pp. 265-282

[21] Biard, T., Le Mauff, A., Bigand, M., and Bourey, J.-P.: 'Separation of decision modeling from business process modeling using new "Decision Model and Notation"(DMN) for automating operational decisionmaking', 'Book Separation of decision modeling from business process modeling using new "Decision Model and Notation"(DMN) for automating operational decision-making' (Springer, 2015, edn.), pp. 489-496

[22] Batoulis, K., Nesterenko, A., Repitsch, G., and Weske, M.: 'Decision Management in the Insurance Industry: Standards and Tools', 'Book Decision Management in the Insurance Industry: Standards and Tools' (2017, edn.), pp. 52-63

[23] OpenRules: 'Rule 2019, https://openrules.com/ruleengine.htm2019

[24] Zaidi, A.K., and Levis, A.H.: 'Validation and verification of decision making rules', Automatica, 1997, 33, (2), pp. 155-169

[25] Hasić, F., De Smedt, J., and Vanthienen, J.: 'Augmenting processes with decision intelligence: Principles for integrated modelling', Decision Support Systems, 2018, 107, pp. 1-12

[26] Amailef, K., and Lu, J.: 'Ontology-supported case-based reasoning approach for intelligent m-Government emergency response services', Decision Support Systems, 2013, 55, (1), pp. 79-97

[27] Osterwalder, A.: 'The business model ontology: A proposition in a design science approach', Institut d'Informatique et Organisation. Lausanne, Switzerland, University of Lausanne, Ecole des Hautes Etudes Commerciales HEC, 2004

[28] Zhao, Y., and Zhu, Q.: 'Evaluation on crowdsourcing research: Current status and future direction', Information Systems Frontiers, 2014, 16, (3), pp. 417-434

[29] Paliwoda-Pękosz, G., and Soja, P.: 'Modeling Enterprise System Adoption: An Ontology-based Approach', AMCIS2014, 2014, Paper 3

[30] Thuan, N.H., Drechsler, A., and Antunes, P.: 'Construction of Design Science Research Questions', Communications of the Association for Information Systems, 2019, 44, (1), pp. 332-363 